Pacific Journal of Mathematic

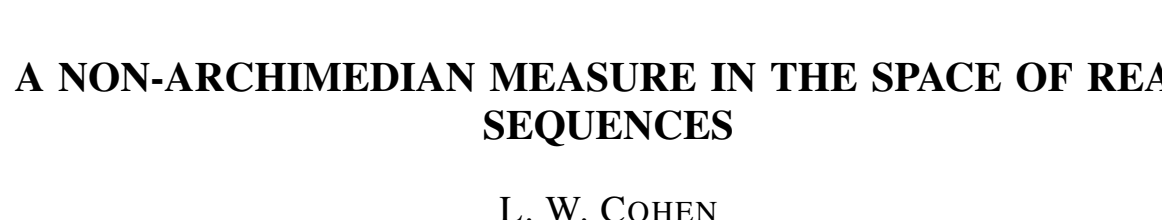




\section{A NON-ARCHIMEDIAN MEASURE IN THE SPACE OF REAL SEQUENCES}

L. W. COHEN

1. Introduction. Let $S$ be the set of real sequences $X=\left(x_{n}\right)$. For $X, Y \in S$ we define $X+Y=\left(x_{n}+y_{n}\right), 0$ as the sequence $x_{n}=0$ and introduce order by writing $X>0$ when for some $m, x_{n}=0$ for $n<m$ and $x_{m}>0$. Thus $S$ may be considered as an ordered abelian group with a nonarchimedian order. Let $S$ be topologized by considering the open intervals

$$
(X, Y)=\{Z \mid X<Z<Y\}
$$

as a basis for the open sets. Then $S$ is a topological group. We note that $S$ is not locally compact. We wish to define a measure on $S$ which is invariant with respect to translations of measurable sets by elements in $S$ and which assigns a nonzero measure to the sets in a basis for the topology in $S$. It is evident from a consideration of the spheres in Hilbert space that such a measure can not in general be real valued for spaces which are not locally compact. In the example studied here the range of the measure function is a subset of $S$.

The ring of measurable sets which serves as the domain of the measure function is generated by a class of sets called intervals. We shall show that these intervals are a basis for the topology of $S$ defined by the open intervals. They have some properties of the real half-open intervals $a^{\prime} \leqq x<a^{\prime \prime}$ which are useful in deriving the properties of a measure function.

For a positive integer $p$ and real numbers

$$
a_{1}, \cdots, a_{p-1}, a_{p}^{\prime}, a_{p}^{\prime \prime}
$$

let $I_{p}=I\left(a_{1}, \cdots, a_{p-1} ; a_{p}^{\prime}, a_{p}^{\prime \prime}\right)$ be the set of $X=\left(x_{n}\right) \in S$ such that

$$
\begin{array}{cr}
x_{n}=a_{n}, & \text { for } n<p, \\
a_{n}^{\prime} \leqq x_{p}<a_{p}^{\prime \prime} & \\
-\infty<x_{n}<+\infty, & n>p .
\end{array}
$$

If $p=1$ there are no conditions on the $x_{n}$ for $n<p$. If $a_{p}^{\prime \prime} \leqq a_{p}^{\prime}$ then $I_{p}$ is empty. That the sets $I_{p}$ and the open intervals $(X, Y)$ are equivalent as bases for neighborhood topologies is shown as follows:

Received September 21, 1954. This paper was written during tenure of a fellowship in 1952-53 from the Fund for Faculty Fellowships, in residence at the Institute for Advanced Study. 
Consider

$$
X=\left(x_{n}\right) \in I\left(a_{1}, \cdots, a_{p-1} ; a_{p}^{\prime}, a_{p}^{\prime \prime}\right)
$$

Then

$$
x_{n}=a_{n} \text {, for } n<p \text {, and } a_{p}^{\prime} \leqq x_{p}<a_{p}^{\prime \prime} \text {. }
$$

Now consider $X_{n}^{\prime}=\left(x_{n}^{\prime}\right), X^{\prime \prime}=\left(x_{n}^{\prime \prime}\right)$ where

$$
\begin{array}{cc}
x_{n}^{\prime}=x_{n}=x_{n}^{\prime \prime} & \text { for } n \leqq p, \\
x_{p+1}^{\prime}<x_{p+1}<x_{p+1}^{\prime \prime} . &
\end{array}
$$

Clearly

$$
\begin{gathered}
X^{\prime}, X^{\prime \prime} \in I\left(a_{1}, \cdots, a_{p-1} ; a_{p}^{\prime}, a_{p}^{\prime \prime}\right), \\
X^{\prime}<X<X^{\prime \prime} .
\end{gathered}
$$

Now if $Y=\left(y_{n}\right) \in\left(X^{\prime}, X^{\prime \prime}\right)$ then

$$
\begin{array}{cc}
x_{n}^{\prime}=y_{n}=x_{n}^{\prime \prime}=a_{n} & \text { for } n<p, \\
a_{p}^{\prime} \leqq x_{p}^{\prime}=x_{p}=y_{p}=x_{p}^{\prime \prime}<a_{p}^{\prime \prime} &
\end{array}
$$

and so $Y \in I\left(a_{1}, \cdots, a_{p-1} ; a_{p}^{\prime}, a_{p}^{\prime \prime}\right)$. Hence

$$
X \in\left(X^{\prime}, X^{\prime \prime}\right) \subset I\left(a_{1}, \cdots, a_{p-1} ; a_{p}^{\prime}, a_{p}^{\prime \prime}\right) .
$$

Conversely, consider $X=\left(x_{n}\right) \in\left(X^{\prime}, X^{\prime \prime}\right)$ where $X^{\prime}=\left(x_{n}^{\prime}\right)<X^{\prime \prime}=\left(x_{n}^{\prime \prime}\right)$. From the definition of order in $S$ it follows that there is an integer $p$ such that

$$
x_{n}^{\prime}=x_{n}=x_{n}^{\prime \prime} \text { for } n<p, x_{p}^{\prime}<x_{p}^{\prime \prime}
$$

and one of the following is true:

(1) $x_{p}^{\prime}<x_{p}<x_{p}^{\prime \prime}$,

(2) $x_{p}^{\prime}<x_{p}=x_{p}^{\prime \prime}$,

(3) $x_{p}^{\prime}=x_{p}<x_{p}^{\prime \prime}$.

If (1) is true let

$$
a_{n}=x_{n} \text { for } n<p, a_{p}^{\prime}=x_{p}, \quad a_{p}^{\prime \prime}=x_{p}^{\prime \prime} .
$$

It follows that

$$
X \in I\left(a_{1}, \cdots, a_{p-1} ; a_{p}^{\prime}, a_{p}^{\prime \prime}\right) \subset\left(X^{\prime}, X^{\prime \prime}\right) .
$$

Suppose (2) is true. Since $X<X^{\prime \prime}$, there is a smallest integer $q>p$ such that $x_{q}<x_{q}^{\prime \prime}$. Now let

$$
a_{n}=x_{n} \text { for } n<q, a_{q}^{\prime}=x_{q} \text { and } a_{q}^{\prime \prime}=x_{q}^{\prime \prime} \text {. }
$$


It follows that

$$
X \in I\left(a_{1}, \cdots, a_{q-1} ; a_{q}^{\prime}, a_{q}^{\prime \prime}\right) \subset\left(X^{\prime}, X^{\prime \prime}\right) .
$$

Suppose (3) is true. Since $X^{\prime}<X$, there is a smallest integer $q>p$ such that $x_{q}^{\prime}<x_{q}$. Let

$$
a_{n}=x_{n} \text { for } n<q, a_{q}^{\prime}=x_{q}, a_{q}^{\prime \prime}=x_{q}+1 .
$$

Again it follows that

$$
X \in I\left(a_{1}, \cdots, a_{q-1} ; a_{q}^{\prime}, a_{q}^{\prime \prime}\right) \subset\left(X^{\prime}, X^{\prime \prime}\right) .
$$

The equivalence of the two bases is established.

For each interval $I_{p}$ the element $\left(x_{n}\right) \in S$ where

$$
x_{p}=\max \left[a_{p}^{\prime \prime}-a_{p}^{\prime}, 0\right] \text { and } x_{n}=0 \text { if } n \neq p
$$

is called the length of $I_{p}$ and is denoted by $\mu\left(I_{p}\right)$. Clearly $\mu\left(I_{p}\right) \geqq 0$ in $S$ and the equality holds if and only if $I_{p}$ is empty. It will be shown that: The intervals $I_{p}$ generate a ring over which the function $\mu$ can be extended to an additive, nonnegative function with values in $S$. If $M$ is a set in the ring and $X+M$ is the set of $X+Y$ for $Y \in M$ then $\mu(M)=\mu(X+M)$. The function $\mu$ may be called an invariant measure on the ring.

2. Properties of Intervals $I_{p}$. Consider two intervals

$$
I_{p}=I\left(a_{1}, \cdots, a_{p-1} ; a_{p}^{\prime}, a_{p}^{\prime \prime}\right), \quad I_{q}=I\left(b_{1}, \cdots, b_{q-1} ; b_{q}^{\prime}, b_{q}^{\prime \prime}\right) .
$$

The following two lemmas are immediate consequences of the definition of interval.

LEMMA 1. $0 \neq I_{q} \subset I_{p}$ if and only if $p \leqq q$, and $a_{n}=b_{n}, \quad n<p$,

$$
\begin{array}{cc}
a_{p}^{\prime} \leqq b_{p}<a_{p}^{\prime \prime}, & p<q, \\
a_{p}^{\prime} \leqq b_{p}^{\prime}<b_{p}^{\prime \prime} \leqq a_{p}^{\prime \prime}, & p=q .
\end{array}
$$

Lemma 2. If $p<q$ and $I_{p} \cap I_{q} \neq 0$ then $I_{q} \subset I_{p}$.

Proof. Since $p<q$ and there is some $X=\left(x_{n}\right) \in I_{p} \cap I_{q}$, we have

$$
\begin{array}{cl}
a_{n}=x_{n}=b_{n}, & n<p, \\
a_{p}^{\prime} \leqq x_{p}=b_{p}<a_{p}^{\prime \prime} . &
\end{array}
$$

It follows from Lemma 1 that $I_{q} \subset I_{p}$. 
Lemma 3. If $I_{p} \cap I_{q} \neq 0$ then $I_{p} \cap I_{q}=I_{r}$ where $r=\max [p, q]$.

LEMMA 4. The union of a finite number of intervals is the union of a finite number of disjoint intervals.

Proof. The statement is true for a single interval. Assume that the statement is true for the union of any $m$ intervals. Consider

$$
I_{p_{i}}, i=1, \cdots, m+1 .
$$

If the intervals (1) are disjoint the statement is true for them. Suppose that for $h \neq j, I_{p_{h}} \cap I_{p_{j}} \neq 0$. If $p_{h}<p_{j}$ then, by Lemma $2, I_{p_{j}} \subset I_{p_{h}}$. Then the intervals (1) have the same union as some $m$ of them and the statement follows from the assumption. If $p_{h}=p_{j}=p$ then, since $I_{p_{h}} \cap I_{p_{j}} \neq 0$, we have

$$
I_{p_{h}}=I\left(a_{1}, \cdots, a_{p-1} ; a_{p}^{\prime}, a_{p}^{\prime \prime}\right), \quad I_{p_{j}}=I\left(a_{1}, \cdots, a_{p-1} ; b_{p}^{\prime}, b_{p}^{\prime \prime}\right),
$$

and the real half open intervals $\left[a_{p}^{\prime}, a_{p}^{\prime \prime}\right),\left[b_{p}^{\prime}, b_{p}^{\prime \prime}\right)$ have a nonempty intersection. If

$$
c_{p}^{\prime}=\min \left(a_{p}^{\prime}, b_{p}^{\prime}\right), c_{p}^{\prime \prime}=\max \left(a_{p}^{\prime \prime}, b_{p}^{\prime \prime}\right)
$$

then $\left[a_{p}^{\prime}, a_{p}^{\prime \prime}\right) \cup\left[b_{p}^{\prime}, b_{p}^{\prime \prime}\right)=\left[c_{p}^{\prime}, c_{p}^{\prime \prime}\right)$ and

$$
I_{p_{h}} \cup I_{p_{k}}=I\left(a_{1}, \cdots, a_{p-1} ; c_{p}^{\prime}, c_{p}^{\prime \prime}\right)=I_{p} .
$$

The intervals (1) have the same union as the $m$ intervals $I_{p}, I_{p_{i}}$ where $i \neq h, j$, and the statement again follows from the assumption. Induction completes the proof.

LEMMA 5. If $I_{p_{i}}, i=1, \cdots, m$, are disjoint nonempty subintervals of $I_{p}$ and $I_{p}=\bigcup_{i=1}^{m} I_{p_{i}}$ then $p_{i}=p$ for $i=1, \cdots, m$, and

$$
\mu\left(I_{p}\right)=\sum_{i=1}^{m} \mu\left(I_{p_{i}}\right) \text {. }
$$

Proof. Let

$$
\begin{gathered}
I_{p}=I\left(a_{1}, \cdots, a_{p-1} ; a_{p}^{\prime}, a_{p}^{\prime \prime}\right) \\
I_{p_{i}}=I\left(a_{i 1}, \cdots, a_{i, p_{i}-1} ; a_{p_{i}}^{\prime}, a_{p_{i}}^{\prime \prime}\right), \quad i=1, \cdots, m .
\end{gathered}
$$

Since $0 \neq I_{p_{i}} \subset I_{p}$, we have $p \leqq p_{i}$, and

$$
\begin{array}{cr}
a_{i n}=a_{n}, & n<p, i=1, \cdots, m, \\
a_{p}^{\prime} \leqq a_{i p}<a_{p}^{\prime \prime}, & p_{i}>p, \\
a_{p}^{\prime} \leqq a_{p_{i}}^{\prime}<a_{p_{i}}^{\prime \prime} \leqq a_{p}^{\prime \prime}, & p_{i}=p .
\end{array}
$$


Consider the half-open intervals $\left[a_{p_{i}}^{\prime}, a_{p_{i}}^{\prime \prime}\right)$ for $p_{i}=p$ and the numbers $a_{i p}$ for $p_{i}>p$. Let $c_{1}, \cdots, c_{k}$ be the distinct numbers among those $a_{i p}$. Since $\bigcup_{i=1}^{m} I_{p_{i}}=I_{p}$ and the $I_{p_{i}}$ are disjoint,

$$
\left[a_{p}^{\prime}, a_{p}^{\prime \prime}\right)=\left(\bigcup_{p_{i}=p}\left[a_{p_{i}}^{\prime}, a_{p_{i}}^{\prime \prime}\right)\right) \cup\left(\bigcup_{j=1}^{k}\left[c_{j}\right]\right)
$$

and the summands are disjoint sets. But a half-open real interval is not such a union unless there are no sets $\left[c_{j}\right]$ consisting of single points. Hence $p_{i}=p$ for $i=1, \cdots, m$ and

$$
a_{p}^{\prime \prime}-a_{p}^{\prime}=\sum_{i=1}^{m}\left(a_{p_{i}}^{\prime \prime}-a_{p_{i}}^{\prime}\right) \text {. }
$$

If $\mu\left(I_{p}\right)=\left(x_{n}\right), \mu\left(I_{p_{i}}\right)=\left(x_{i n}\right)$ then, since $p_{i}=p$ and $I_{p_{i}} \neq 0$,

$$
\begin{array}{lr}
x_{n}=x_{i n}=0, & n \neq p, i=1, \cdots, m, \\
x_{p}=a_{p}^{\prime \prime}-a_{p}^{\prime}, & \\
x_{i p}=a_{p_{i}}^{\prime \prime}-a_{p_{i}}^{\prime}, & i=1, \cdots, m,
\end{array}
$$

and it follows from (1) that

$$
\sum_{i=1}^{m} \mu\left(I_{p_{i}}\right)=\left(\sum_{i=1}^{m} x_{i n}\right)=\left(x_{n}\right)=\mu\left(I_{p}\right) .
$$

Lemma 6. If $I_{p_{i}}, i=1, \cdots, m$, and $J_{q_{j}}, j=1, \cdots, n$, are two sets of disjoint intervals with the same union then

$$
\sum_{i=1}^{m} \mu\left(I_{p_{i}}\right)=\sum_{j=i}^{n} \mu\left(J_{p_{j}}\right) \text {. }
$$

Proof. Since, by Lemma 2, the intersection of two intervals is an interval, possibly empty, the sets $I_{p_{i}} \cap J_{\alpha_{j}}$ are disjoint intervals. Since the $I_{p_{i}}$ and the $J_{q_{j}}$ have the same union, we have

$$
\begin{array}{ll}
I_{p_{i}}=\bigcup_{j=1}^{n}\left(I_{p_{i}} \cap J_{q_{j}}\right), & i=1, \cdots, m, \\
J_{q_{j}}=\bigcup_{i=1}^{m}\left(I_{p_{i}} \cap J_{q_{j}}\right), & j=1, \cdots, n .
\end{array}
$$

Applying Lemma 5 and recalling that $\mu\left(I_{p}\right)=0 \in S$ if $I_{p}$ is empty, we obtain

$$
\begin{aligned}
& \mu\left(I_{p_{i}}\right)=\sum_{j=1}^{n} \mu\left(I_{p_{i}} \cap J_{q_{j}}\right), \\
& \mu\left(J_{q_{j}}\right)=\sum_{i=1}^{m} \mu\left(I_{p_{i}} \cap J_{q_{j}}\right) .
\end{aligned}
$$


Since $S$ is an abelian group,

$$
\sum_{i=1}^{m} \mu\left(I_{p_{i}}\right)=\sum_{i=1}^{m} \sum_{j=1}^{n} \mu\left(I_{p_{i}} \cap J_{q_{j}}\right)=\sum_{j=1}^{n} \mu\left(J_{q_{j}}\right) .
$$

In order to obtain properties of differences of unions of intervals

$$
\bigcup_{i=1}^{m} I_{p_{i}}-\bigcup_{j=1}^{n} J_{q_{j}}
$$

it will be sufficient to consider the special class $\mathscr{D}$ of sets

$$
E=I_{p}-\bigcup_{i=1}^{m} I_{p_{i}}
$$

$$
I_{p_{i}} \text { disjoint, } I_{p_{i}} \subset I_{p}, i=1, \cdots, m \text {. }
$$

Since $I_{p_{i}} \subset I_{p}$, either $p_{i} \geqq p$ or $I_{p_{i}}=0 .^{1} \mathrm{~A}$ set $E \in \mathscr{D}$ is called proper if, among the $I_{p}, I_{p_{i}}$ used to represent it, $p_{i}>p$.

LEMma 7. If $E \in \mathscr{D}$ then $E$ is the union of a finite number of disjoint proper elements of $\mathscr{D}$.

Proof. If $E \in \mathscr{D}$ then

$$
E=I_{p}-\bigcup_{i=1}^{m} I_{p_{i}}
$$

where

$$
\begin{aligned}
I_{p} & =I\left(a_{1}, \cdots, a_{p-1} ; a_{p}^{\prime}, a_{p}^{\prime \prime}\right), \\
I_{p_{i}} & =I\left(a_{i 1}, \cdots, a_{i, p_{i}-1} ; a_{p_{i}}^{\prime}, a_{p_{i}}^{\prime \prime}\right), \quad i=1,2, \cdots, m,
\end{aligned}
$$

and the $I_{p_{i}}$ are disjoint subsets of $I_{p}$. Hence $p_{i} \geqq p$ and $a_{i n}=a_{n}$ for $n<p$. If $p_{i}=p$ then $\sigma_{i}=\left[a_{p_{i}}^{\prime}, a_{p_{i}}^{\prime \prime}\right) \subset\left[a_{p}^{\prime}, a_{p}^{\prime \prime}\right)=\sigma$ and the $\sigma_{i}$ are disjoint.

$$
\sigma-\bigcup_{p_{i}=p} \sigma_{i}=\bigcup_{j=1}^{h} \tau_{j}
$$

where the $\tau_{j}=\left[b_{j}^{\prime}, b_{j}^{\prime \prime}\right)$ are disjoint. Let

$$
I_{p}^{j}=I\left(a_{1}, \cdots, a_{p-1} ; b_{j}^{\prime}, b_{j}^{\prime \prime}\right), \quad \alpha_{j}=\left\{i \mid a_{i_{p}} \in \tau_{j} \text { and } p_{i}>p\right\}, \quad j=1, \cdots, h .
$$

The $\alpha_{j}$ are disjoint; and $I_{p_{i}} \subset I_{p}^{j}$ if and only if $p_{i}>p$ and $i \in \alpha_{j}$. The sets

$$
E_{j}=I_{p}^{j}-\bigcup_{i \in a_{j}} I_{p_{i}} \quad j=1, \cdots, h,
$$

${ }^{1}$ It will be assumed that the $I_{p_{i}}$ in a representation of a set $E$ are not empty. This does not sacrifice any generality. 
are disjoint proper elements of $\mathscr{D}$ whose union is $E$. This is so because

$$
I_{p}-\bigcup_{p=p_{i}} I_{p_{i}}=\bigcup_{j=1}^{n} I_{p}^{j}
$$

and every $I_{p_{i}}$ with $p_{i}>p$ is in some $I_{p}^{j}$.

LEMMA 8. If

$$
E=I_{p}-\bigcup_{i=1}^{m} I_{p_{i}}, \quad F=J_{p}-\bigcup_{j=1}^{n} J_{q_{j}}
$$

are proper sets in $\mathscr{D}$ then $E \cap F=0$ if and only if $I_{p} \cap J_{p}=0$.

Proof. Since $E \subset I_{p}, F \subset J_{p}$ it is clear that $E \cap F=0$ if $I_{p} \cap J_{p}=0$. Suppose $I_{p} \cap J_{p} \neq 0$. Let

$$
\begin{aligned}
& I_{p}=I\left(a_{1}, \cdots, a_{p-1} ; a_{p}^{\prime}, a_{p}^{\prime \prime}\right), J_{p}=I\left(b_{1}, \cdots, b_{p-1} ; b_{p}^{\prime}, b_{p}^{\prime \prime}\right), \\
& I_{p_{i}}=I\left(a_{i 1}, \cdots, a_{i, p_{i}-1} ; a_{p_{i}}^{\prime}, a_{p_{i}}^{\prime \prime}\right), J_{p_{j}}=I\left(b_{j 1}, \cdots, b_{j, p_{j}-1} ; b_{p_{j}}^{\prime}, b_{p_{j}}^{\prime \prime}\right), \\
& i=1, \cdots, m, j=1, \cdots, n .
\end{aligned}
$$

Since $E$ and $F$ are proper, $p_{i}, q_{j}>p$. Since $I_{p} \cap J_{p} \neq 0$, we have $a_{n}=b_{n}, n<p$, and $\left[a_{p}^{\prime}, a_{p}^{\prime \prime}\right) \cap\left[b_{p}^{\prime}, b_{p}^{\prime \prime}\right)=\left[c^{\prime}, c^{\prime \prime}\right) \neq 0$. The half-open interval $\left[c^{\prime}, c^{\prime \prime}\right)$ contains a number $x \neq a_{i p}, b_{j p}, i=1, \cdots, m, j=1, \cdots, n$. If $X=\left(x_{n}\right)$ where $x_{p}=x$ and $x_{n}=a_{n}$, for $n<p$, then $X \in E \cap F$. Hence if $E \cap F=0$ then $I_{p} \cap J_{p}=0$.

For

$$
E=I_{p}-\bigcup_{i=1}^{m} I_{p_{i}} \in \mathscr{D}
$$

we define $\mu(E) \in S$ by

$$
\mu(E)=\mu\left(I_{p}\right)-\sum_{i=1}^{m} \mu\left(I_{p_{i}}\right) .
$$

It is to be noted that a set $E$ may have two representations;

$$
E=I_{p}-\bigcup_{i=1}^{m} I_{p_{i}}=J_{q}-\bigcup_{j=1}^{n} J_{q_{j}}
$$

and the uniqueness of $\mu(E)$ must be proved (cf. corollary to Lemma 11). In order to do this and to prove the additivity of $\mu$ as a function on $\mathscr{D}$ to $S$ we make some definitions which are useful.

If

$$
I_{p}=I\left(a_{1}, \cdots, a_{p-1} ; a_{p}^{\prime}, a_{p}^{\prime \prime}\right)
$$

we call $p$ the rank of $I_{p}, a_{n}$ the $n$th point component of $I_{p}$ and $\left[a_{p}^{\prime}, a_{p}^{\prime \prime}\right)$ 
the interval component of $I_{p}$. Given a set of nonempty intervals $I_{p_{i}}, \cdots$, $I_{p_{m}}$ the number $N$ of distinct ranks $p_{i}$ is called the spread of the set of intervals. For example, if $E$ is a proper set in $\mathscr{D}$, then the spread of $E$ is 1 if and only if $E$ is an interval $I_{p}$.

LeMma 9. If

(a) $I_{p_{i}}, i=1, \cdots, m$, are nonempty, disjoint intervals,

(b) $E_{j}=J_{q_{j}}-\bigcup_{k=1}^{k_{j}} J_{q_{j k}}, j=1, \cdots, h$, are nonempty, disjoint, proper sets in $\mathscr{D}$,

then

(c) $\bigcup_{i \rightarrow 1}^{m} I_{p_{i}}=\bigcup_{j=1}^{h} E_{j}$

$$
\sum_{i=1}^{m} \mu\left(I_{p_{i}}\right)=\sum_{j=1}^{n} \mu\left(E_{j}\right)
$$

Proof. Let $N$ be the spread of the set of intervals $I_{p_{i}}, J_{q_{j}}, J_{q_{j k}}$. If $N=1, p_{i}=q_{j}=p$ and the sets $E_{j}$ are the intervals $J_{q_{j}}$ since the $E_{j}$ are proper. The conclusion follows from Lemma 6 .

Assume that $N>1$ and that the lemma is proved if the spread of the set of intervals in (a), (b) is $N-1$.

First we show that if $p=\min \left(p_{1}, \cdots, p_{m}\right), q=\min \left(q_{1}, \cdots, q_{h}\right)$ then $p=q$. Suppose $p<q$. There is some $p_{r}=p$. The $p$ th component of $I_{p_{r}}$ is a half-open interval $\sigma$ and the pth component of $J_{q_{j}}$ is a point $b_{j}$. There is a number $x \in \sigma-\left\{b_{1}, \cdots, b_{h}\right\}$. If $X=\left(x_{n}\right)$ where $x_{p}=x$ and $x_{n}, n<p$, is the $n$th component of $I_{p_{r}}$ then

$$
X \in I_{p_{r}}-\bigcup_{j=1}^{n} J_{q_{j}} \subset \bigcup_{i=1}^{m} I_{p_{i}}-\bigcup_{j=1}^{n} E_{j}
$$

contrary to (c). Hence $q \leqq p$. Suppose $q<p$. There is some $q_{r}=q$ and

$$
E_{r}=J_{q_{r}}-\bigcup_{k=1}^{k_{r}} J_{q_{r k}} \neq 0, \quad q_{r k}>q_{r} .
$$

The $q$ th component of $J_{q_{r}}$ is a nonempty half-open interval $\tau$, the $q$ th components of $J_{q_{r k}}, k=1, \cdots, k_{r}$, and of $I_{p_{i}}$ are points, say $c_{1}, \cdots, c_{s}$. There is a number $x \in \tau-\left\{c_{1}, \cdots, c_{s}\right\}$. If $X=\left(x_{n}\right)$ where $x_{q}=x$ and $x_{n}$, $n<q$, is the $n$th component of $J_{q_{r}}$,

$$
X \in\left(J_{q_{r}}-\bigcup_{k=1}^{k_{r}} J_{q_{r k}}\right)-\bigcup_{i=1}^{m} I_{p_{i}} \subset \bigcup_{j=1}^{h} E_{j}-\bigcup_{i=1}^{m} I_{p_{i}},
$$

contrary to (c). Hence $p=q$.

Next, we show that

$$
\bigcup_{p_{i}=p} I_{p_{i}}=\bigcup_{q_{j}=p} J_{q_{j}} .
$$


Let

$$
A^{\prime}=\bigcup_{p_{i}=p} I_{p_{i}}, \quad A^{\prime \prime}=\bigcup_{q_{j}=p} J_{q_{j}} .
$$

Suppose $A^{\prime \prime}-A^{\prime} \neq 0$. For some $q_{r}=p$, there is

$$
X=\left(x_{n}\right) \in J_{q_{r}}-\bigcup_{p_{i}=p} I_{p_{i}} \text {. }
$$

Let

$$
\begin{aligned}
\sigma & =\text { the interval component of } J_{q_{r}}, \\
\sigma_{i} & =\text { the interval component of } I_{p_{i}} \text { where } p_{i} \approx p, \\
\alpha & =\left\{i \mid J_{q_{r}} \cap I_{p_{i}} \neq 0 \text { and } p_{i}=p\right\} .
\end{aligned}
$$

Then

$$
x_{p} \in \sigma-\bigcup_{i \in \infty} \sigma_{i}
$$

and so there is a nonempty, half-open interval $\tau$ such that

$$
\tau \subset \sigma-\bigcup_{i \in \infty} \sigma_{i}
$$

The $p$ th components of the $I_{p_{i}}, p_{i}>p$, and of $J_{q_{r k}}, k=1, \cdots, k_{r}$ are finite in number, say $c_{1}, \cdots, c_{s}$. Hence there is a number $y$ such that

$$
y \in \tau-\left\{c_{1}, \cdots, c_{s}\right\} \text {. }
$$

If $Y=\left(y_{n}\right)$ where $y_{p}=y$ and $y_{n}, n<p$, is the $n$th component of $J_{q_{r}}$,

$$
Y \in\left(J_{q_{r}}-\bigcup_{k=1}^{k} J_{q_{r k}}\right)-\bigcup_{i=1}^{m} I_{p_{i}} \subset \bigcup_{j=1}^{n} E_{j}-\bigcup_{i=1}^{m} I_{p_{i}},
$$

contrary to (c). A similar argument shows that $A^{\prime}-A^{\prime \prime} \neq 0$ leads to a contradiction. Hence (1) is proved.

Since the $E_{j}$ are disjoint proper sets in $\mathscr{D}$ it follows from Lemma 8 that $I_{q_{r}} \cap I_{q_{s}}=0$ if $p=q_{r}=q_{s}$ and $r \neq s$. Hence, from (1) and Lemma 6,

$$
\sum_{p_{i}=p} \mu\left(I_{p_{i}}\right)=\sum_{q_{j}=p} \mu\left(J_{q_{j}}\right) \text {. }
$$

From (c) and (1)

$$
\left(\bigcup_{p_{i}>p} I_{p_{i}}\right) \cup\left(\bigcup_{q_{j}=p} J_{q_{j}}\right)=\left(\bigcup_{q_{j}=p}\left(J_{q_{j}}-\bigcup_{k=1}^{k_{j}} J_{q_{j k}}\right)\right) \cup\left(\bigcup_{q_{j}>p} E_{j}\right) .
$$

It follows from (a), (1) that the two unions on the left are disjoint and from (b) that the two unions on the right are disjoint. Hence

$$
\left(\bigcup_{p_{i}>p} I_{p_{i}}\right) \cup\left(\bigcup_{q_{j}=p} \bigcup_{k=1}^{k_{j}} J_{q_{j k}}\right)=\bigcup_{q_{j}>p} E_{j} \text {. }
$$


The ranks of the intervals $I_{p_{i}}, J_{q_{j}}, J_{q_{j k}}$ occurring in (4) exclude $p$ since $p_{i}>p, q_{j k}>q_{j}=p$ on the left and $q_{j k}>q_{j}>p$ on the right. Hence the spread of the set of intervals in (4) is $N-1$. Since the $E_{j}$ are disjoint it follows from Lemma 8 that the $J_{q_{j}}, q_{j}=p$, are disjoint. Since for each $j$, the $J_{q_{j k}}$ are disjoint in $k$ and $J_{q_{j k}} \subset J_{q_{j}}$, the $J_{q_{j k}}$ are disjoint in $j, k$ for $q_{j}=p$. It follows from (1), (a) that the intervals on the left of (4) are disjoint. Thus the set of nonempty intervals on the left of (4) satisfy (a) of the lemma, the set of $E_{j}$ on the right satisfy (b), and (4) is (c) for the intervals involved. Since the spread is $N-1$, we have, by the assumption of the lemma for $N-1$,

$$
\sum_{p_{i}>p} \mu\left(I_{p_{i}}\right)+\sum_{q_{j}=p} \sum_{k=1}^{k_{j}} \mu\left(J_{q_{j k}}\right)=\sum_{q_{j}>p} \mu\left(E_{j}\right) \text {. }
$$

Combining (2), (5), it follows that

$$
\begin{aligned}
\sum_{i=1}^{m} \mu\left(I_{p_{i}}\right) & =\sum_{p_{l}>p} \mu\left(I_{p_{i}}\right)+\sum_{p_{i}=p} \mu\left(I_{p_{i}}\right)=\sum_{q_{j}=p}\left(\mu\left(J_{q_{j}}\right)-\sum_{k=1}^{k_{j}} \mu\left(J_{q_{j k}}\right)\right)+\sum_{q_{j}>p} \mu\left(E_{j}\right) \\
& =\sum_{q_{j}=p} \mu\left(E_{j}\right)+\sum_{q_{j}>p} \mu\left(E_{j}\right)=\sum_{j=1}^{h} \mu\left(E_{j}\right) .
\end{aligned}
$$

Lemma 10. For $E \in \mathscr{D}, \mu(E)=0 \in S$ if $E$ is empty and

$$
\mu(E)=\sum_{j=1}^{n} \mu\left(E_{j}\right)
$$

if $E=\bigcup_{j=1}^{n} E_{j}$ where the $E_{j}$ are nonempty, disjoint, proper sets in $\mathscr{D}$.

Proof. If $E \in \mathscr{D}$, then

$$
E=I_{p}-\bigcup_{i=1}^{m} I_{p_{i}}
$$

where the $I_{p_{i}}$ are disjoint subsets of $I_{p}$. If $E$ is empty, then

$$
\bigcup_{i=1}^{m} I_{p_{i}}=I_{p}
$$

and it follows from Lemma 5 that

$$
\mu(E)=\mu\left(I_{p}\right)-\sum_{i=1}^{m} \mu\left(I_{p_{i}}\right)=0 \in S .
$$

If $E=\bigcup_{j=1}^{n} E_{j}$ where the $E_{j}$ are nonempty, disjoint, proper sets in $\mathscr{D}$ then

$$
I_{p}=\left(\bigcup_{j=1}^{n} E_{j}\right) \cup\left(\bigcup_{i=1}^{m} I_{p_{i}}\right)
$$

and the intervals in the set $\left\{I_{p}, E_{j}, I_{p_{i}} \neq 0\right\}$ satisfy the conditions of 
Lemma 9. Since $\mu\left(I_{p_{i}}\right)=0$ if $I_{p_{i}}$ is empty, it follows that

$$
\begin{aligned}
& \mu\left(I_{p}\right)=\sum_{j=1}^{n} \mu\left(E_{j}\right)+\sum_{i=1}^{m} \mu\left(I_{p_{i}}\right), \\
& \mu(E)=\mu\left(I_{p}\right)-\sum_{i=1}^{m} \mu\left(I_{p_{i}}\right)=\sum_{j=1}^{n} \mu\left(E_{j}\right) .
\end{aligned}
$$

Lemma 11. If $E \in \mathscr{D}$ and $E_{1}, \cdots, E_{m}$ are disjoint elements of $\mathscr{D}$ such that

$$
E=\bigcup_{i=1}^{m} E_{i}
$$

then

$$
\mu(E)=\sum_{i=1}^{m} \mu\left(E_{i}\right)
$$

Proof. It follows from Lemma 10 that the statement is true if $E=0$ and that if $E \neq 0$ only $E_{i} \neq 0$ need be considered. By Lemma 7,

$$
E_{i}=\bigcup_{j=1}^{j_{i}} E_{i j}
$$$$
i=1, \cdots, m
$$

where the $E_{i j}, j=1, \cdots, j_{i}$, are disjoint, nonempty, proper elements of $\mathscr{D}$. Since the $E_{i}$ are disjoint, the $E_{i j}$ are disjoint in $i, j$. Now

$$
E=\bigcup_{i=1}^{m} \bigcup_{j=1}^{j_{i}} E_{i j}
$$

By Lemma 10,

$$
\mu(E)=\sum_{i=1}^{m} \sum_{j=1}^{j_{i}} \mu\left(E_{i j}\right)=\sum_{i=1}^{m} \mu\left(E_{i}\right)
$$

Corollary. For $E \in \mathscr{D}, \mu(E)$ is unique.

This follows from Lemma 11 with $m=1$.

Lemma 12. For $E \in \mathscr{D}, \mu(E) \geqq 0$ in the order in $S$.

Proof. If $E=0, \mu(E)=0$. If $E$ is a nonempty, proper set in $\mathscr{D}$ then

$$
E=I_{p}-\bigcup_{i=1}^{m} I_{p_{i}}
$$

and $p_{i}>p$. Now $\mu\left(I_{p}\right)=\left(x_{n}\right), \mu\left(I_{p_{i}}\right)=\left(x_{i n}\right), i=1, \cdots, m$, and 


$$
\begin{array}{rr}
x_{p}>0, \quad x_{n}=0, & n \neq p \\
x_{i n}=0, & n \leqq p<p_{i}, i=1, \cdots, m .
\end{array}
$$

Since

$$
\begin{gathered}
\mu(E)=\mu\left(I_{p}\right)-\sum_{i=1}^{m} \mu\left(I_{p_{i}}\right)=\left(x_{n}-\sum_{i=1}^{m} x_{i m}\right), \\
x_{n}-\sum_{i=1}^{m} x_{i n}=0 \\
x_{p}-\sum_{i=1}^{m} x_{i p}=x_{p}>0
\end{gathered}
$$

it follows that $\mu(E)>0$ in the order in $S$.

It now follows from Lemmas 7, 11 and the fact that the sum of positive elements of $S$ is positive that $\mu(E) \geqq 0$ for $E \in \mathscr{D}$.

3. On Generating a Ring. The set of intervals $I_{p}$, having the properties of Lemmas 2, 4 is an example of a class $\mathscr{C}$ of sets satisying the following conditions :

(i) $0 \in \mathscr{C}$.

(ii) If $A, B \in \mathscr{C}$ then $A \cap B \in \mathscr{C}$.

(iii) If $A_{1}, \cdots, A_{m} \in \mathscr{C}$ there are disjoint $B_{1}, \cdots, B_{n} \in \mathscr{C}$ such that

$$
\bigcup_{i=1}^{m} A_{i}=\bigcup_{j=1}^{n} B_{j} .
$$

Let $\mathscr{D}$ be the class of sets $E$ such that

(iv) $E=A-\bigcup_{i=1}^{m} A_{i}, A, A_{i} \in \mathscr{C}, A_{i}$ disjoint, $A_{i} \subset A$.

Let $\mathscr{R}$ be the class of sets $M$ such that

(v) $M=\bigcup_{i=1}^{m} E_{i}, E_{i} \in \mathscr{D}, E_{i}$ disjoint.

We note that $\mathscr{C} \subset \mathscr{D} \subset \mathscr{R}$. It will be shown that $\mathscr{R}$ is a ring.

Lemma 13. If $E, F \in \mathscr{D}$ then $E \cap F \in \mathscr{D}$.

Proof. There are sets $A, A_{i}, B, B_{j}$ satisfying (iv) such that

$$
E=A-\bigcup_{i=1}^{m} A_{i}, \quad F=B-\bigcup_{j=1}^{n} B_{j} .
$$

Now

$$
E \cap F=A \cap B-\left(\bigcup_{j=1}^{n}\left(A \cap B_{j}\right)\right) \cup\left(\bigcup_{i=1}^{m}\left(A_{j} \cap B\right)\right) .
$$

By (ii), $A \cap B, A \cap B_{j}, A_{i} \cap B$ are in $\mathscr{C}$. It follows from (iii) that there are disjoint $C_{1}, \cdots, C_{s} \in \mathscr{C}$ such that 


$$
\left(\bigcup_{j=1}^{n}\left(A \cap B_{j}\right)\right) \cup\left(\bigcup_{i=1}^{m}\left(A_{i} \cap B\right)\right)=\bigcup_{k=1}^{s} C_{k} .
$$

Since $C_{k} \subset A \cap B$ and

$$
E \cap F=A \cap B-\bigcup_{k=1}^{s} C_{k},
$$

we have $E \cap F \in \mathscr{D}$.

Lemma 14. $E, F \in \mathscr{D}$ there are disjoint $E_{0}, \cdots, E_{s} \in \mathscr{D}$ such that

$$
E-F=\bigcup_{k=0}^{s} E_{k}
$$

Proof. There are $A, A_{i}, B, B_{j} \in \mathscr{C}$ satisfying (iv) such that

$$
E=A-\bigcup_{i=1}^{m} A_{i}, \quad F=B-\bigcup_{j=1}^{n} B_{j} .
$$

Let

$$
E_{0}=(A-A \cap B) \cap E, \quad E_{j}=B_{j} \cap E, \quad j=1, \cdots, n .
$$

Now $A-A \cap B \in \mathscr{D}$ and it follows from Lemma 13 that $E_{j} \in \mathscr{D}, j=0$, $\cdots, n$. Since $E_{0} \cap B=0, E_{j} \subset B_{j} \subset B$ and the $B_{j}, j=1, \cdots, n$, are disjoint, $E_{0}, E_{1}, \cdots, E_{n}$ are disjoint. From

$$
\bigcup_{j=0}^{n} E_{j} \subset E
$$

and

$$
E_{0} \cap F \subset(A-A \cap B) \cap B=0, \quad E_{j} \cap F \subset B_{j} \cap F=0, \quad j=1, \cdots, n
$$

follows

$$
\bigcup_{j=0}^{n} E_{j} \subset E-F
$$

On the other hand

$$
\begin{gathered}
E-F \subset\left(A-\bigcup_{i=1}^{m} A_{i}\right)-\left(B-\bigcup_{j=1}^{n} B_{j}\right) \subset(A-A \cap B) \cap E \cup\left(\bigcup_{j=1}^{n}\left(B_{j} \cap E\right)\right) \\
=\bigcup_{j=0}^{n} E_{j} .
\end{gathered}
$$

Hence

$$
E-F=\bigcup_{j=0}^{n} E_{\jmath}, \quad E_{\jmath} \in \mathscr{D}, \quad E_{\jmath} \text { disjoint. }
$$


Theorem 1. F is a ring.

Proof. For $M, N \in \mathscr{R}$ there are disjoint sets $E_{i} \in \mathscr{Z}$ and disjoint sets $F_{j} \in \mathscr{D}$ such that

$$
M=\bigcup_{i=1}^{m} E_{i}, \quad N=\bigcup_{j=1}^{n} F_{j} .
$$

The sets $E_{i} \cap F_{j}$ are disjoint and, by Lemma 13, belong to $\mathscr{D}$. Hence

$$
M \cap N=\left(\bigcup_{i=1}^{m} E_{i}\right) \cap\left(\bigcup_{j=1}^{n} F_{j}\right)=\bigcup_{i=1}^{m} \bigcup_{j=1}^{n}\left(E_{\imath} \cap F_{j}\right) \in \mathscr{R} .
$$

Now

$$
\begin{aligned}
M-M \cap N & =\bigcup_{i=1}^{m} E_{i}-\bigcup_{i=1}^{m} \bigcup_{j=1}^{n}\left(E_{i} \cap F_{j}\right)=\bigcup_{i=1}^{m}\left(E_{i}-E_{i} \cap\left(\bigcup_{j=1}^{n} F_{j}\right)\right) \\
& =\bigcup_{i=1}^{m} \bigcap_{j=1}^{n}\left(E_{i}-E_{i} \cap F_{j}\right) .
\end{aligned}
$$

By Lemma 14, $M_{i, j}=E_{i}-E_{i} \cap F_{j}$ is the union of a finite number of disjoint sets in $\mathscr{C}$ and so $M_{i, j} \in$ It follows from (1) that

$$
M_{i}=\bigcap_{j=1}^{n} M_{i j} \in \mathscr{R}, \quad i=1, \cdots, m .
$$

Since each $M_{i} \subset E_{i}$ and the $E_{i}$ are disjoint, the $M_{i}$ are disjoint. Each $M_{i}$ is the union of a finite number of disjoint sets in $\varnothing$. Hence

$$
M-M \cap N=\bigcup_{i=1}^{m} M_{i} \in \mathscr{R} \text {. }
$$

Finally,

$$
M \cup N=(M-M \cap N) \cup(M \cap N) \cup(N-M \cap N) \text {. }
$$

It follows from (1), (2) that each summand is in $\mathscr{R}$. Since the summands are disjoint and are the unions of disjoint sets in $\mathscr{Z}$,

$$
M \cup N \in \Re \text {. }
$$

That $\mathscr{R}$ is a ring follows from (1), (2), (3).

4. The Measure Function on $\mathscr{R}_{2}$ to The function $\mu\left(I_{\eta}\right)$ on the class $\sigma^{2}$ of intervals $I_{p}$ to $S$ is extended to a function on $C$ to $S$ which is additive and nonnegative in the sense of the corollary to Lemma 11 and Lemma 12. If $M$ is in the ring $\mathscr{R}$ of unions of disjoint sets in is then

$$
M=\bigcup_{i=1}^{m} E_{i}
$$


where the $E_{i}$ are disjoint sets in $\mathscr{D}$. We define

$$
\mu(M)=\sum_{i=1}^{m} \mu\left(E_{i}\right)
$$

THEOREM 2. $\mu(M)$ is a single valued function on $\mathscr{R}$ to $S$ such that $\mu(M) \geq 0$ and

$$
\mu(M)=\sum_{i=1}^{m} \mu\left(M_{i}\right) \text { if } M=\bigcup_{i=1}^{m} M_{i}, M_{i} \in \mathscr{R}, \quad M_{i} \text { disjoint } .
$$

Proof. Suppose

$$
M=\bigcup_{i=1}^{m} E_{i}=\bigcup_{j=1}^{n} F_{j}
$$

where the sets $E_{i}$ and the sets $F_{j}$ are disjoint elements of $\mathscr{Q}$. Then

$$
\begin{array}{ll}
E_{i}=\bigcup_{j=1}^{n}\left(E_{i} \cap F_{j}\right), & i=1, \cdots, m, \\
F_{j}=\bigcup_{i=1}^{m}\left(E_{i} \cap F_{j}\right), & j=1, \cdots, n,
\end{array}
$$

and the disjoint sets $E_{i} \cap F_{j}$ are elements of $\mathscr{D}$ by Lemma 13. From Lemma 11,

$$
\begin{aligned}
& \mu\left(E_{i}\right)=\sum_{j=1}^{n} \mu\left(E_{i} \cap F_{j}\right), \\
& \mu\left(F_{j}\right)=\sum_{i=1}^{m} \mu\left(E_{i} \cap F_{j}\right) .
\end{aligned}
$$

Since $S$ is an abelian group,

$$
\mu(M)=\sum_{i=1}^{m} \mu\left(E_{i}\right)=\sum_{i=1}^{m} \sum_{j=1}^{n} \mu\left(E_{i} \cap F_{j}\right)=\sum_{j=1}^{n} \mu\left(F_{j}\right) .
$$

Hence $\mu(M)$ is a single valued function on $\mathscr{R}$ to $S$.

Since $\mu(E) \geqq 0$ in $S$ for $E \in \mathscr{D}$ and the sum of nonnegative elements in $S$ is nonnegative, we have $\mu(M) \geqq 0$ in $\mathscr{R}$.

If $M=\bigcup_{i=1}^{m} M_{i}$ and the $M_{i}$ are disjoint elements in $\mathscr{R}$,

$$
M_{i}=\bigcup_{j=1}^{n_{i}} E_{i j}
$$

$i=1, \cdots, m$,

and

$$
M=\bigcup_{i=1}^{m} \bigcup_{j=1}^{n_{i}} E_{i j}
$$

where the $E_{i j}$ are disjoint elements in $\mathscr{D}$. Hence 


$$
\mu(M)=\sum_{i=1}^{m} \sum_{j=1}^{n_{i}} \mu\left(E_{i j}\right)=\sum_{i=1}^{m} \mu\left(M_{i}\right)
$$

Theorem 3. If $M \in \mathscr{R}, X \in S$ and

$$
X+M=\{X+Y \mid Y \in M\}
$$

then $X+M \in \mathscr{R}$ and $\mu(X+M)=\mu(M)$.

Proof. If $I_{p}=I\left(a_{1}, \cdots, a_{p-1} ; a_{p}^{\prime}, a_{p}^{\prime \prime}\right)$ and $X=\left(x_{n}\right)$ then

$$
X+I_{p}=I\left(x_{1}+a_{1}, \cdots, x_{p-1}+a_{p-1}, x_{p}+a_{p}^{\prime}, x_{p}+a_{p}^{\prime \prime}\right) \in \mathscr{C} \subset \mathscr{R}
$$

and

$$
\mu\left(X+I_{p}\right)=\mu\left(I_{p}\right)
$$

If

$$
M=E=I_{p}-\bigcup_{i=1}^{m} I_{p_{i}} \in \mathscr{D},
$$

then

$$
X+M=\left(X+I_{p}\right)-\bigcup_{i=1}^{m}\left(X+I_{p_{i}}\right) \in \mathscr{D} \subset \mathscr{R}
$$

and, by (1),

$$
\mu(X+M)=\mu\left(I_{p}\right)-\sum_{i=1}^{m} \mu\left(I_{p_{i}}\right)=\mu(M) .
$$

If $M=\bigcup_{i=1}^{m} E_{i}$ and the $E_{i}$ are disjoint sets in $\mathscr{D}$, then $X+E_{i}$ are disjoint sets in $\mathscr{D}$ and, by $(2), \mu\left(E_{i}\right)=\mu\left(X+E_{i}\right)$. Since

$$
X+M=\bigcup_{i=1}^{m}\left(X+E_{i}\right) \in \mathscr{R},
$$

we have

$$
\mu(X+M)=\sum_{i=1}^{m} \mu\left(X+E_{i}\right)=\sum_{i=1}^{m} \mu\left(E_{i}\right)=\mu(M) .
$$

The following observations were suggested by O. Nikodým, to whom the author is indebted for a helpful reading of the manuscript. Given $X=\left(x_{n}\right) \in S$ such that all but a finite number of the $x_{n}$ are zero, there is a measurable $M \in \mathscr{R}$ such that $\mu(M)=X$. The results obtained here for real valued sequences (over the ordinals $n<\omega$ ) may be extended by the same methods to the space of real valued sequences $x_{\alpha}$ over any given initial section of ordinals $\alpha<\xi$. 


\section{PACIFIC JOURNAL OF MATHEMATICS}

\section{EDITORS}

H. L. Royden

Stanford University

Stanford, California

E. HewitT

University of Washington

Seattle 5 , Washington
R. P. Dilworth

California Institute of Technology Pasadena 4, California

E. G. Straus

University of California

Los Angeles 24, California

\section{ASSOCIATE EDITORS}

E. F. BECKENBACH

C. E. BURGESS

H. BUSEMANN

H. FEDERER

\author{
M. HALL \\ P. R. HALMOS \\ V. GANAPATHY IYER \\ R. D. JAMES
}

M. S. KNEBELMAN

I. NIVEN

T. G. OSTROM

M. M. SCHIFFER
J. J. STOKER

G. SZEKERES

F. WOLF

K. YOSIDA

\section{SUPPORTING INSTITUTIONS}

UNIVERSITY OF BRITISH COLUMBIA

CALIFORNIA INSTITUTE OF TECHNOLOGY

UNIVERSITY OF CALIFORNIA

MONTANA STATE UNIVERSITY

UNIVERSITY OF NEVADA

OREGON STATE COLLEGE

UNIVERSITY OF OREGON

UNIVERSITY OF SOUTHERN CALIFORNIA
STANFORD UNIVERSITY

UNIVERSITY OF UTAH

WASHINGTON STATE COLLEGE

UNIVERSITY OF WASHINGTON

AMERICAN MATHEMATICAL SOCIETY CALIFORNIA RESEARCH CORPORATION HUGHES AIRCRAFT COMPANY 


\section{Pacific Journal of Mathematics}

\section{Vol. 6, No. $1 \quad$ November, 1956}

David Blackwell, An analog of the minimax theorem for vector payoffs..... 1

L. W. Cohen, A non-archimedian measure in the space of real

sequences ..................................... 9

George Bernard Dantzig, Constructive proof of the Min-Max theorem ..... 25

Jim Douglas, On the numerical integration of quasilinear parabolic

differential equations ............................... 35

James Michael Gardner Fell, A note on abstract measure ............. 43

Isidore Isaac Hirschman, Jr., A note on orthogonal systems . . . . . . . . . . 47

Frank Harary, On the number of dissimilar line-subgraphs of a given

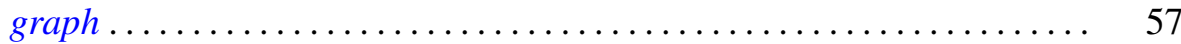

Newton Seymour Hawley, Complex bundles with Abelian group......... 65

Alan Jerome Hoffman, Morris Newman, Ernst Gabor Straus and Olga

Taussky, On the number of absolute points of a correlation ...........

Ernst Gabor Straus and Olga Taussky, Remark on the preceding paper.

Algebraic equations satisfied by roots of natural numbers . . ........ 97

Ralph D. James, Summable trigonometric series ................. 99

Gerald R. Mac Lane, Limits of rational functions . . . . . . . . . . . . . . . 111

F. Oberhettinger, Note on the Lerch zeta function ................. 117

Gerald C. Preston, On locally compact totally disconnected Abelian groups and their character groups ........................... 121

Vikramaditya Singh and W. J. Thron, On the number of singular points, located on the unit circle, of certain functions represented by

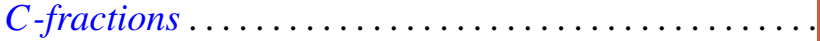

Sherman K. Stein, The symmetry function in a convex body ... 145 Edwin Weiss, Boundedness in topological rings.............

Albert Leon Whiteman, A sum connected with the series for the partition

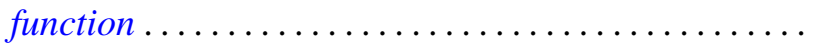

Alfred B. Willcox, Some structure theorems for a class of Banach algebras.

Joseph Lawrence Zemmer, Some remarks on p-rings and their Boolean geometry... 